\title{
Time to buy a squid?
}

from Hilton Stowell

THE past decade has witnessed the evolution of the once esoteric art of 'gross evoked potentials' into the profitable biomedical technology of 'event related brain potentials' (ERBP). The new name is both more accurate and more informative. The essential difference between traditional electroencephalography (EEG) and ERBP is this: Whereas the EEG looked at the electrical activity of the whole brain, for many seconds of time, and in much ignorance of what that particular brain was doing during that time, ERBP examines the electrical activity of cooperating networks of cortical neurones when, and only when, they are responding to specific and controllable inputs or outputs of information.

As often happens in the history of science, technological innovation has improved our appreciation of a theoretical problem. Before the invention of the 'signal averager' there was little understanding of the importance of timing for cerebral events, except perhaps among a handful of psychophysicists; it had been a sufficient achievement, in the time of Hans Berger and Lord Adrian, to make the brainwaves visible without penetrating the skull. Then the computer of average transients spotlighted time, often at the expense of space; the spatial resolution of ERBP is notoriously weak.

Now, perhaps the most significant technical advance since the birth of the 'signal averager' has ocurred. This newcomer is the superconducting quantum

Hilton Stowell is a former Research Scientist at the ERPB Unit, Central State Hosptial of Georgia. interference device (SQUID). Originally a geologist's instrument for detecting very weak magnetic fields, the SQUID has been applied to the analysis of human brainwaves since around the mid-nineteenseventies in the United States. It is now accepted, both in the United States and in Europe, as a necessary adjunct to conventional electric-field recording ${ }^{1-3}$. Why is it useful?

The SQUID attacks ERBP methods at their weakest point, which is the accurate determination of cerebral sources and sinks; and the inference therefrom of cortical dipole origins for specific temporal components of the sequence of voltage polarity reversals which make up an 'evoked potential' of the brain. Its ability to do this ${ }^{4-6}$ depends, in the first place, on two elementary physical properties of the magnetic field associated with every electric current: First, the magnetic field strength attenuates faster with distance from its origin than does the electric field; second, it is neither attenuated nor smeared by low-conductivity tissues such as the skull. A more trivial advantage of magnetic gradiometry is its obvious ability to do without skin-contact electrodes on the scalp, and without that pervasive nuisance of traditional electroencephalography, the hopefully 'neutral' or 'indifferent' reference electrode on an earlobe, nose-tip, or more distant part of the body which inevitably picks up the ECG also. Moreover, what might seem to be the gravest disadvantage of measuring very weak magntic fields turns out to be yet another advantage over traditional ERBP and EEG. No magnetic shielding is needed in ordinary suburban environments ${ }^{5}$. The asymmetric gradiometry technique is sensitive only to the magnetic field difference between its two linked, asymmetrical loops, while rejecting components of the magnetic field common to both. Of course, like its rough analogue the a.c. differential amplifier, the SQUID's common mode rejection is limited. You don't put your stimulating equipment close to the subject's head, or set up your ERBP laboratory next door to a large magnet. But the magnetic fields of neural generators close to the scalp can be quite sharply localized by a SQUID sitting $1.5 \mathrm{~cm}$ off the scalp.

Now for the disadvantages: the SQUID double-loop must sit within a dewar of liquid helium, and this limits manoeuvreability whilst increasing cost. Worst of all, data must be signal-averaged from only one gradiometer at a time. So scalp topography must be done serially rather than in parallel from a large array of conventional EEG electrodes. The absence of messy scalp-preparation and electrodesticking may bring a slight compensation in time-saving.

At the time of writing there may be halfa-dozen ERBP laboratories systematically using SQUIDs in the United State and perhaps two or three in Europe (one in Finland certainly). I predict that within five years it will be difficult to publish useful 'human evoked potential' data without one.

\footnotetext{
. Reite, M. et al. EEG clin. Neurophysiol. 40, 59 (1976). Reite, M. et al. EEG clin. Neurophysiol. 45, 114 (1978) Reite M\& Zimmerman. J. A Rev. Biophys. Bioengng 7 $167(1978)$.

4. Aittoniemi, K, et al Proc Third Nat Meet. Biophys. Med. Eng. Fintand (Lappecnranta, 1979). Hari, R. et al. Expl Brain Res. 40, 237 (1980). Zimmerman, J.T. et al. EEG clin. Neurophysiol. 52.
} $151(1981)$

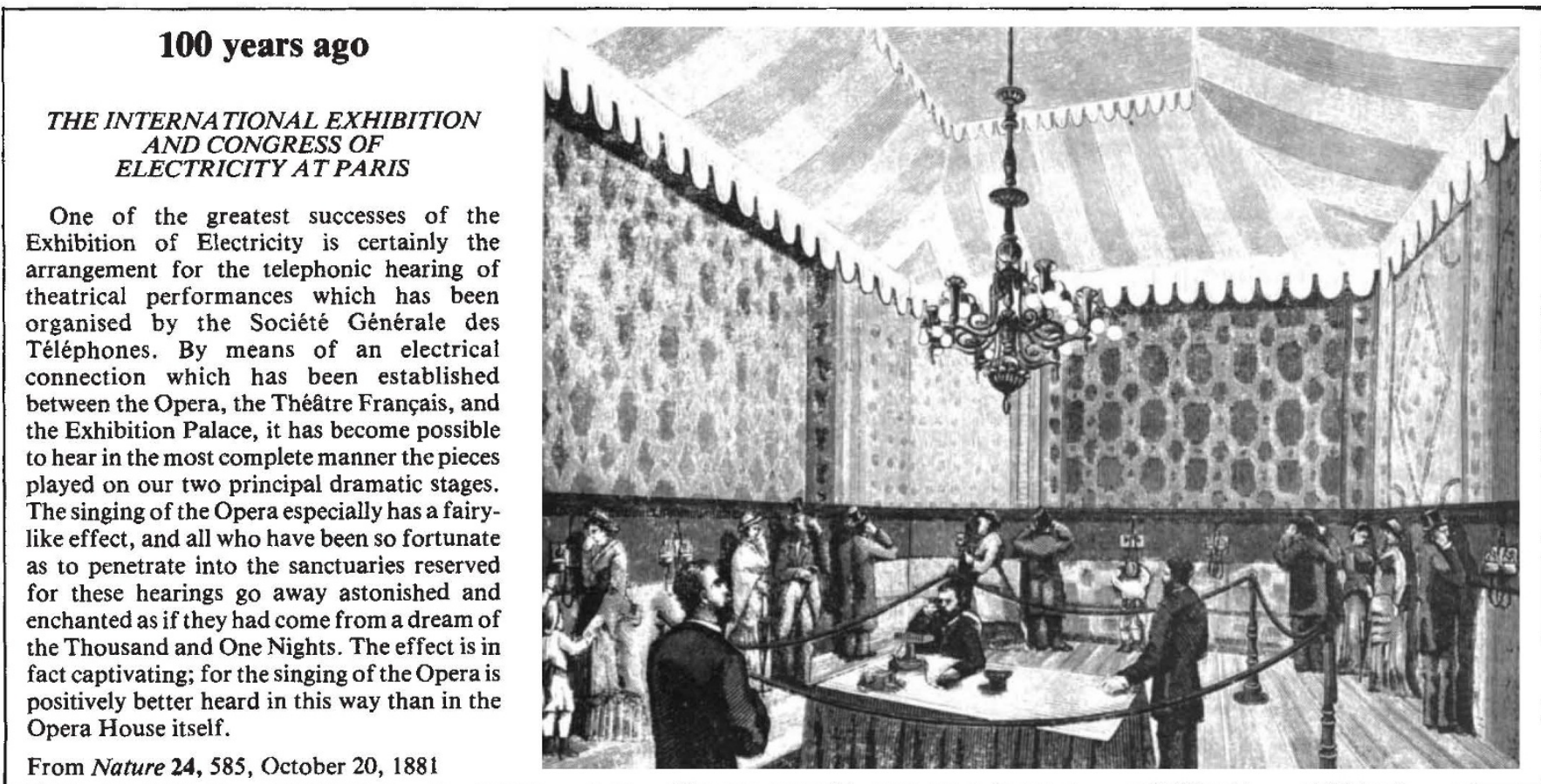

